Geometry $8 \mathcal{F}$ Topology

Volume 3 (1999) 119-135

Published: 29 May 1999

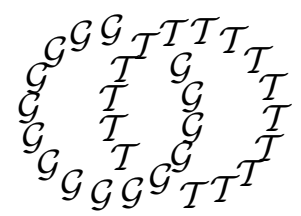

\title{
The bottleneck conjecture
}

\author{
Greg KuperberG \\ Department of Mathematics, University of California \\ One Shields Avenue, Davis, CA 95616, USA \\ Email: greg@math.ucdavis.edu
}

\begin{abstract}
The Mahler volume of a centrally symmetric convex body $K$ is defined as $M(K)=(\operatorname{Vol} K)\left(\operatorname{Vol} K^{\circ}\right)$. Mahler conjectured that this volume is minimized when $K$ is a cube. We introduce the bottleneck conjecture, which stipulates that a certain convex body $K^{\diamond} \subset K \times K^{\circ}$ has least volume when $K$ is an ellipsoid. If true, the bottleneck conjecture would strengthen the best current lower bound on the Mahler volume due to Bourgain and Milman. We also generalize the bottleneck conjecture in the context of indefinite orthogonal geometry and prove some special cases of the generalization.
\end{abstract}

This article is in the $\mathrm{xxx}$ archive as: math.MG/9811119

\section{AMS Classification numbers Primary: 52A40}

Secondary: 46B20, 53C99

Keywords: Metric geometry, euclidean geometry, Mahler conjecture, bottleneck conjecture, central symmetry

Proposed: Robion Kirby

Seconded: Walter Neumann, Yasha Eliashberg
Received: 23 November 1998

Accepted: 20 May 1999

\section{Copyright Geometry and Topology}


Let $V$ be an $n$-dimensional vector space and let $V^{*}$ be the dual vector space. We denote the usual inner product between $V$ and $V^{*}$ by $\langle\cdot, \cdot\rangle$. If $K \subset V$ is a centrally symmetric convex body centered at the origin, then there is a convex body

$$
K^{\circ}=\left\{\vec{y} \in V^{*} \mid\langle K, \vec{y}\rangle \subseteq[-1,1]\right\}
$$

called the dual or polar body of $K$. The Mahler volume of $K$ is defined as

$$
M(K)=\operatorname{Vol} K \times K^{\circ}=(\operatorname{Vol} K)\left(\operatorname{Vol} K^{\circ}\right) .
$$

Here $V$ and $V^{*}$ are given dual volume structures, or for the first expression, the natural volume structure on $V \times V^{*}$ suffices.

The Mahler volume arises in the geometry of numbers and in functional analysis. By construction it is invariant under the action of $\mathrm{GL}(V)$ on $K$. For fixed $V$, the space of symmetric convex bodies divided by the action of $\mathrm{GL}(V)$ is compact in the Hausdorff topology, and $M(K)$ is continuous under this action. Consequently $M(K)$ has a finite maximum and a non-zero minimum in each dimension. The maximum and minimum of $M(K)$ are interesting objects of study in asymptotic convex geometry:

Theorem 1 (Santaló) In a fixed vector space $V, M(K)$ is uniquely maximized by ellipsoids.

Let $C_{n}$ be the standard unit cube and let $B_{n}$ be the round unit ball, both in $\mathbb{R}^{n}$. The polar body $C_{n}^{\circ}$ is the standard cross polytope, while obviously $B_{n}^{\circ}=B_{n}$.

Conjecture 1 (Mahler) For convex bodies $K$ in $n$ dimensions with $n$ fixed, the volume $M(K)$ is minimized by the cube $C_{n}$.

Conjecture 1 is considered harder than Theorem 1 because a cube has much less symmetry than an ellipsoid. Moreover, $M(K)$ cannot be uniquely minimized when $K$ is a cube or a cross polytope, because there are other polytopes with the same Mahler volume. For example,

$$
M\left(C_{a+b}\right)=M\left(C_{a} \times C_{b}^{\circ}\right) .
$$

By contrast, Theorem 1 can be proved by an elegant symmetrization argument [6].

Using methods from functional analysis, Bourgain and Milman [1] proved an asymptotic version of Conjecture 1: 
Theorem 2 (Bourgain, Milman) There is a constant $c>0$ such that for any $n$ and any centrally-symmetric convex body $K$ of dimension $n$,

$$
M(K) \geq c^{n} M\left(B_{n}\right) .
$$

Although the proof technically constructs the constant $c$ (and although the proof has been simplified [5]), no good value for it is currently known. The author [2] proved the following:

Theorem 3 If $K$ has dimension $n \geq 4$, then

$$
M(K) \geq\left(\log _{2} n\right)^{-n} M\left(B_{n}\right) .
$$

Theorem 3 has no arbitrary constants and therefore has some strength in low dimensions, but it is obviously asymptotically weaker than Theorem 2 .

In this paper, we present a conjecture (Conjecture 2 below) which would produce a good value for the constant $c$ in Theorem 2 . The conjecture also motivated the proof of Theorem 3.

Let

$$
\begin{aligned}
& K^{+}=\left\{(\vec{x}, \vec{y}) \in K \times K^{\circ} \mid\langle\vec{x}, \vec{y}\rangle=1\right\} \\
& K^{-}=\left\{(\vec{x}, \vec{y}) \in K \times K^{\circ} \mid\langle\vec{x}, \vec{y}\rangle=-1\right\}
\end{aligned}
$$

and let $K^{\diamond}$ be the convex hull of $K^{+} \cup K^{-}$.

Conjecture 2 For convex bodies $K$ in $n$ dimensions with $n$ fixed the volume

$$
D(K)=\operatorname{Vol} K^{\diamond}
$$

is uniquely minimized when $K$ is an ellipsoid.

We call Conjecture 2 the bottleneck conjecture, because the equation $\langle\vec{x}, \vec{y}\rangle=1$ defines a hyperboloid sheet $H^{+}$in $V \times V^{*}$ that resembles the flange of a bottle, while $K^{+}$is a topological sphere in $H^{+}$that forms a neck. Figure 1 shows the geometry in the trivial case $n=1$, which serves as a schematic for the higher-dimensional case. The inclusion

$$
K^{\diamond} \subseteq K \times K^{\circ}
$$

obviously implies the inequality

$$
D(K) \leq M(K)
$$




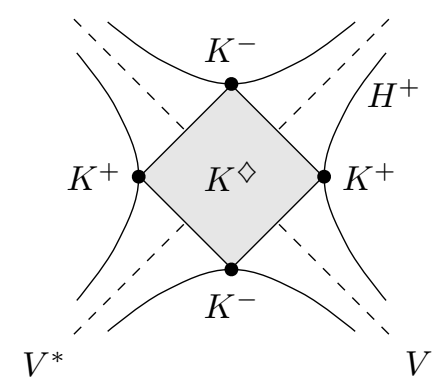

Figure 1: The geometry of $K^{+}, K^{-}$, and $K^{\diamond}$

To see the strength of Conjecture 2, consider these volume formulas:

$$
\begin{aligned}
\operatorname{Vol} C_{n} & =2^{n} & \operatorname{Vol} C_{n}^{\circ} & =\frac{2^{n}}{n !} \\
\operatorname{Vol} B_{n} & =\frac{\pi^{n / 2}}{(n / 2) !} & \frac{M\left(C_{n}\right)}{M\left(B_{n}\right)} & =\frac{(4 / \pi)^{n}}{\left(\begin{array}{c}
n \\
n / 2
\end{array}\right)}
\end{aligned}
$$

(Here $\frac{n}{2} !=\Gamma\left(\frac{n}{2}+1\right)$ when $n$ is half-integral.) The body $B_{n}^{\diamond}$ is the convex hull of two orthogonal round $n$-balls of radius $\sqrt{2}$ in $\mathbb{R}^{2 n}$, so

$$
\operatorname{Vol} B_{n}^{\diamond}=\left(\operatorname{Vol} B_{n}\right)^{2} \frac{2^{n}}{\left(\begin{array}{c}
2 n \\
n
\end{array}\right)} \text {. }
$$

Consequently, if $\left\{K_{n}\right\}$ is any sequence of symmetric convex bodies with $\operatorname{dim} K_{n}$ $=n$, then Conjecture 1 implies that

$$
\lim _{n \rightarrow \infty} \sqrt[n]{\frac{M\left(K_{n}\right)}{M\left(B_{n}\right)}} \geq \frac{2}{\pi}
$$

if the limit exists, while Conjecture 2 implies that

$$
\lim _{n \rightarrow \infty} \sqrt[n]{\frac{M\left(K_{n}\right)}{M\left(B_{n}\right)}} \geq \frac{1}{2}
$$

if the limit exists.

\section{Reformulations}

The main purpose of this section is to introduce another conjecture which implies Conjecture 2 and which may be equivalent. 
Conjecture 3 If $K \subset V$ is a centrally symmetric convex body, then

$$
Q\left(\overrightarrow{\mathrm{Vol}} K^{+}\right)
$$

the energy of the directed volume enclosed by $K^{+}$, is uniquely minimized when $K$ is an ellipsoid.

Here is an explanation of the terminology of Conjecture 3 . The space $W=$ $V \times V^{*}$ has a symmetric bilinear form extending the pairing of $V$ and $V^{*}$ and such that

$$
\langle\vec{x}, \vec{y}\rangle=0
$$

if $\vec{x}$ and $\vec{y}$ are both in $V$ or both in $V^{*}$. (There is an even more important antisymmetric, or symplectic, form that extends the pairing, but in this article the symmetric extension is the relevant one.) The function $Q$ is the associated quadratic form on $W$ given by

$$
Q(\vec{v})=\langle\vec{v}, \vec{v}\rangle
$$

These forms have signature $(n, n)$, where $n$ is the dimension of $V$. Both the inner product and the quadratic form extend to the exterior algebra $\wedge^{*} W$ by the relation

$$
Q\left(\omega_{1} \wedge \omega_{2}\right)=Q\left(\omega_{1}\right) Q\left(\omega_{2}\right) .
$$

In this paper the quantity $Q(\omega)$ is called the energy of the tensor $\omega$. The energy form $Q$ on the space $\bigwedge^{k} W$ of $k$-tensors has signature

$$
\left(\frac{a+b}{2}, \frac{a-b}{2}\right)
$$

where

$$
a=\left(\begin{array}{c}
2 n \\
k
\end{array}\right) \quad b= \begin{cases}(-1)^{k}\left(\begin{array}{c}
n \\
k / 2
\end{array}\right) & k \text { even } \\
0 & k \text { odd }\end{cases}
$$

If $M \subset W$ is an oriented smooth $k$-manifold with boundary, it has a directed volume

$$
\overrightarrow{\mathrm{Vol}} M \in \bigwedge^{k} W .
$$

If $M$ is the image of a smooth embedding

$$
\vec{f}: U \rightarrow W
$$

of some domain $U \subset \mathbb{R}^{k}$, then the directed volume is given by an integral formula:

$$
\overrightarrow{\mathrm{Vol}} M=\int_{U} d \vec{f}=\int_{U} \frac{\partial \vec{f}}{\partial x_{1}} \wedge \frac{\partial \vec{f}}{\partial x_{2}} \wedge \ldots \wedge \frac{\partial \vec{f}}{\partial x_{k}} d \vec{x} .
$$


By Stokes' theorem, $\overrightarrow{\mathrm{Vol}} M$ only depends on the boundary of $M$. If $N$ is an oriented, closed $(k-1)$-manifold, we define the directed volume $\overrightarrow{\mathrm{Vol}} N$ enclosed by $N$ as the directed volume of any oriented $M$ with $\partial M=N$.

\subsection{Conjecture 3 implies Conjecture 2}

The point of Conjecture 3 is that the energy of the directed volume of $K$ is, up to a constant factor, the volume of the region $K^{\diamond} \subset K^{\diamond}$ enclosed by line segments that connect $K^{+}$to $K^{-}$. The bodies $K^{\diamond}$ and $K^{\diamond}$ could be identical for all $K$. We will develop some geometric properties of $K^{-}$and $K^{+}$to argue that

$$
Q\left(\overrightarrow{\mathrm{Vol}} K^{+}\right)
$$

is essentially an integral formula for the volume of $K^{\odot}$.

A vector $\vec{v} \in W$ is spacelike if $Q(\vec{v})>0$, timelike if $Q(\vec{v})<0$, and null if $Q(\vec{v})=0$. A manifold in $W$ is spacelike if all tangent vectors are spacelike; it is timelike if all tangent vectors are timelike. There is a principle of transversality of space and time: If $V^{+}$is a spacelike vector subspace of $W$ and $V^{-}$is a timelike vector subspace, then

$$
V^{-} \cap V^{+}=\{\overrightarrow{0}\} .
$$

Thus, any basis of $V^{+}$and any basis of $V^{-}$are linearly independent in $W$.

Let $H^{+}$and $H^{-}$be the hypersurfaces defined by

$$
H^{ \pm}=\left\{\vec{v} \mid Q(\vec{v})= \pm \frac{1}{2}\right\} .
$$

Both hypersurfaces are diffeomorphic to $\mathbb{R}^{n} \times S^{n-1}$. Pick some ellipsoid $E \subset V$ centered at the origin. Then $E$ determines a self-adjoint isomorphism

$$
\phi: V \rightarrow V^{*}
$$

such that

$$
E=\{\vec{x} \in V \mid\langle\vec{x}, \phi(\vec{x})\rangle \leq 1\} .
$$

Let $V^{+}$and $V^{-}$be the $n$-planes in $W$ defined by

$$
V^{ \pm}=\{(\vec{x}, \pm \phi(\vec{x}))\} \text {. }
$$

Then

$$
E^{ \pm}=V^{ \pm} \cap H^{ \pm}
$$

The linear space $V^{+}$is spacelike, while $V^{-}$is timelike. The projection of $H^{+}$ onto $V^{+}$along $V^{-}$consists of all points of $V^{+}$except those enclosed by $E^{+}$. 
The composition of this linear projection with radial projection onto $E^{+}$is a convenient map

$$
\pi^{+}: H^{+} \rightarrow E^{+}
$$

to $E^{+}$, which is a topological $(n-1)$-sphere. Each fiber $\pi^{-1}(\vec{v})$ of this map is a timelike section of $H^{+}$which is isometric to hyperbolic $n$-space.

As before, let $K$ be a symmetric convex body in $V$. For simplicity, assume that both $K$ and $K^{\circ}$ are smooth. For each point $\vec{x} \in \partial K$, there is a unique $\vec{y} \in \partial K^{\circ}$, the outward normal of $\partial K$ at $\vec{x}$, such that

$$
\langle\vec{x}, \vec{y}\rangle=1 .
$$

Moreover, for each such $\vec{x}$, the body $K$ has an osculating ellipsoid $E(\vec{x})$, defined as the unique ellipsoid with the following three properties:

(1) $\vec{x}$ lies in $\partial E(\vec{x})$.

(2) $\vec{y}$ is the outward normal of $E(\vec{x})$ at $\vec{x}$.

(3) $\partial E(\vec{x})$ has the same extrinsic curvature as $\partial K$ at $\vec{x}$.

Equivalently, $E(\vec{x})^{+}$and $K^{+}$have the same tangent $(n-1)$-plane at the point $(\vec{x}, \vec{y})$. The existence of $E(\vec{x})$ for each $\vec{x}$ implies that $K^{+}$is a spacelike manifold, that is, that its tangent spaces are spacelike. In fact, for each $\vec{v} \in K^{+}$, the $n$-plane spanned by $T_{\vec{v}} K^{+}$and $\vec{v}$ is spacelike. Finally, the restriction of the projection $\pi^{+}$to $K^{+}$is a homeomorphism between $K^{+}$and $B_{n}^{+}$.

Let $J=K^{+} * K^{-}$be the topological join of $K^{+}$and $K^{-}$. Explicitly,

$$
J=\left(K^{+} \times K^{-} \times[0,1]\right) / \sim
$$

where the equivalence relation $\sim$ is given by

$$
\left(\vec{x}, \vec{y}_{1}, 0\right) \sim\left(\vec{x}, \vec{y}_{2}, 0\right) \quad\left(\vec{x}_{1}, \vec{y}, 1\right) \sim\left(\vec{x}_{2}, \vec{y}, 1\right) .
$$

There is a natural map

$$
\vec{\jmath}: J \rightarrow W
$$

defined by

$$
\vec{\jmath}(\vec{x}, \vec{y}, t)=t \vec{x}+(1-t) \vec{y}
$$

In the following proposition and below, the adverb almost means "up to a set of measure 0".

Proposition 1 The map $\vec{\jmath}$ is almost a smooth embedding. The set $\vec{\jmath}(J)$ meets almost every ray from the origin in $W$ exactly once. 
Proof Let $S_{W}$ be the space of such rays, and let

$$
\pi_{W}: J \rightarrow S_{W}
$$

be the composition of $\vec{\jmath}$ with radial projection to $S_{W}$. The space $J$ is a smooth manifold except on $K^{+}$and $K^{-}$, where it is merely a Lipschitz manifold. Let $\vec{x} \in K^{+}$and $\vec{y} \in K^{-}$. By the space-time transversality principle, the vectors and tangent spaces $\vec{x}, T_{\vec{x}} K^{+}, \vec{y}$, and $T_{\vec{y}} K^{-}$are linearly independent. Thus, the map $\pi$ has positive Jacobian at each point $(\vec{x}, \vec{y}, t) \in J$ with $0<t<1$, because the derivative matrix can be explicitly expressed in terms of $\vec{x}, \vec{y}$, and bases for $T_{\vec{x}} K^{+}$and $T_{\vec{y}} K^{-}$. In other words, $\pi$ is a local diffeomorphism away from $K^{+}$and $K^{-}$. The map $\pi$ is Lipschitz on $K^{+}$and $K^{-}$themselves, which implies that $\pi_{W}\left(K^{+}\right)$and $\pi_{W}\left(K^{-}\right)$are sets of measure zero.

The degree of the map $\pi_{W}$ is both an integer and continuous as a function of $K$. It follows that the degree is 1 , since that is its value when $K$ is an ellipsoid. Thus $\pi$ is almost a diffeomorphism, as desired.

We conjecture that $\pi_{W}$ is a homeomorphism (without excepting a set of measure zero).

As mentioned above, $K^{\odot}$ is defined as the region in $W$ enclosed by $\vec{\jmath}(J)$. By Proposition $1, K^{\odot}$ is almost starlike.

Let $\vec{x} \in K^{+}$and let $P(\vec{x})$ be a tangent infinitesimal parallelepiped at $\vec{x}$. Let $\vec{y} \in$ $K^{-}$and define $P(\vec{y})$ likewise. Let $P(\vec{x}, \vec{y})$ be the semi-infinitesimal polytope which is the convex hull of $P(\vec{x}), P(\vec{y})$, and the origin. If the directed volume of $P(\vec{x})$ is $d \vec{x}$ and the directed volume of $P(\vec{y})$ is $d \vec{y}$, then the volume of $P(\vec{x}, \vec{y})$ is

$$
\frac{1}{\left(\begin{array}{c}
2 n \\
n
\end{array}\right)} \vec{x} \wedge \vec{y} \wedge d \vec{x} \wedge d \vec{y}
$$

The body $K^{\odot}$ is disjoint union of all $P(\vec{x}, \vec{y})$ as $\vec{x}$ and $\vec{y}$ vary, and by Proposition 1, they are almost disjoint. Consequently

$$
\operatorname{Vol} K^{\odot}=\int_{K^{+}} \int_{K^{-}} \frac{1}{\left(\begin{array}{c}
2 n \\
n
\end{array}\right)} \vec{x} \wedge \vec{y} \wedge d \vec{x} \wedge d \vec{y} .
$$

This equation factors as

$$
\left(\begin{array}{c}
2 n \\
n
\end{array}\right) \operatorname{Vol} K^{\odot}=\left(\int_{K^{+}} \vec{x} \wedge d \vec{x}\right) \wedge\left(\int_{K^{-}} \vec{y} \wedge d \vec{y}\right) .
$$

Let $L^{+}$be the union of line segments from $K^{+}$to the origin and let $L^{-}$be the analogous cone over $K^{-}$. Then

$$
\overrightarrow{\mathrm{Vol}} K^{ \pm}=\overrightarrow{\mathrm{Vol}} L^{ \pm}=\int_{K^{ \pm}} \vec{x} \wedge d \vec{x}
$$


by decomposition into infinitesimal cones. Thus, equation (1) further simplifies to

$$
\begin{aligned}
\left(\begin{array}{c}
2 n \\
n
\end{array}\right) \operatorname{Vol} K^{\odot} & =\left(\overrightarrow{\mathrm{Vol}} L^{+}\right) \wedge\left(\overrightarrow{\mathrm{Vol}} L^{-}\right) \\
& =\left(\overrightarrow{\mathrm{Vol}} K^{+}\right) \wedge\left(\overrightarrow{\mathrm{Vol}} K^{-}\right) .
\end{aligned}
$$

Finally, the linear map

$$
\sigma: W \rightarrow W
$$

defined by

$$
\sigma(\vec{x}, \vec{y})=(-\vec{x}, \vec{y})
$$

for $\vec{x} \in V$ and $\vec{y} \in V^{*}$ sends $K^{+}$to $K^{-}$and negates the quadratic form $Q$. Both $\sigma$ and $Q$ extend to the exterior algebra $\Lambda^{*} W$. Functoriality of directed volume then implies that

$$
\overrightarrow{\mathrm{Vol}} K^{-}=\sigma \overrightarrow{\mathrm{Vol}} K^{+}
$$

If $\vec{e}_{1}, \ldots, \vec{e}_{n}$ is a basis for $V$, and if $\phi$ is a self-adjoint isomorphism from $V$ to $V^{*}$ (as defined previously), then

$$
\vec{e}_{1}+\phi\left(\vec{e}_{1}\right), \vec{e}_{2}+\phi\left(\vec{e}_{2}\right), \ldots, \vec{e}_{n}+\phi\left(\vec{e}_{n}\right)
$$

is a basis for $V^{+}$(also defined previously). Then because $\phi$ is self-adjoint, the wedge product

$$
\omega=\left(\vec{e}_{1}+\phi\left(\vec{e}_{1}\right)\right) \wedge\left(\vec{e}_{2}+\phi\left(\vec{e}_{2}\right)\right) \wedge \ldots \wedge\left(\vec{e}_{n}+\phi\left(\vec{e}_{n}\right)\right)
$$

satisfies the identity

$$
\langle\omega, \nu\rangle=\sigma(\omega) \wedge \nu
$$

for an arbitrary $n$-tensor $\nu$. (It is easy to verify this identity with an explicit calculation in the representative case where $V$ is $\mathbb{R}^{n}$ with the standard basis and $\phi$ is the identity.) Because of the system of osculating ellipsoids for $K$, and because of equation (2), $\overrightarrow{\mathrm{Vol}} K^{+}$is a linear combination of such tensors $\omega$, which means that it satisfies equation (5) as well. In particular,

$$
\overrightarrow{\mathrm{Vol}} K^{+} \wedge \sigma\left(\overrightarrow{\mathrm{Vol}} K^{+}\right)=Q\left(\overrightarrow{\mathrm{Vol}} K^{+}\right) .
$$

Combining this identity with equations (3) and (4) yields

$$
\left(\begin{array}{c}
2 n \\
n
\end{array}\right) \operatorname{Vol} K^{\odot}=Q\left(\overrightarrow{\operatorname{Vol}} K^{+}\right)
$$

Since $K^{\odot}$ is always contained in $K^{\diamond}$, and since they coincide when $K$ is an ellipsoid, this final expression shows that Conjecture 3 implies Conjecture 2, as desired. 


\subsection{A generalization}

There is a plausible generalization of Conjecture 3 to $a+b$ dimensions, by which we mean a vector space $V$ with an inner product of signature $(a, b)$. Let $Q$ be the associated quadratic form. Let

$$
H^{+}=\{\vec{x} \in V \mid Q(\vec{x})=1\}
$$

be the positive unit hyperboloid sheet associated to $Q$. (Note that $H^{+}$is now slightly different, because it was previously the level set $Q^{-1}(1 / 2)$.) Also for convenience endow $V$ with a volume form relative to which the inner product has determinant $(-1)^{b}$.

Conjecture 4 Let $H^{+}$be the positive unit hyperboloid of a non-singular quadratic form $Q$ on a vector space $V$ with signature $(a, b)$. Let $N$ be a spacelike submanifold of $\mathrm{H}^{+}$whose inclusion into $\mathrm{H}^{+}$is a homotopy equivalence. Then $Q(\overrightarrow{\mathrm{Vol}} N)$, the energy of the directed volume enclosed by $N$, is uniquely minimized when $N$ is the intersection of $Q$ with an $a$-plane in $V$ containing the origin.

Call a manifold $N$ as defined in Conjecture 4 a neck. Conjecture 3 is the special case of Conjecture 4 when $a=b$, and only for those necks which can be realized as $K^{+}$for some convex body $K$.

We could even more generally ask to minimize the inner product

$$
\left\langle\overrightarrow{\mathrm{Vol}} N_{1}, \overrightarrow{\mathrm{Vol}} N_{2}\right\rangle
$$

for two different spacelike necks $N_{1}$ and $N_{2}$. Or we could minimize the wedge product

$$
\overrightarrow{\mathrm{Vol}} N^{+} \wedge \overrightarrow{\mathrm{Vol}} N^{-}
$$

for a spacelike neck $N^{+}$in $H^{+}$and a timelike neck $N^{-}$in $H^{-}$. (The wedge product can be interpreted as a number using the volume form on $V$.) In the author's opinion, Conjecture 4 is a natural starting point for this family of questions.

\section{Proofs in marginally indefinite cases}

In this section we will prove Conjecture 4 in the four least indefinite cases: $1+n, n+1, n+2$, and $2+n$ dimensions. Note that in an $(a+b)$-dimensional 
vector space $V$, the set of spacelike $a$-planes is contractible, so we can consistently orient them. Likewise we can consistently orient timelike $b$-planes. For convenience, we choose orientations which are consistent with the orientation of $V$ induced by its volume form.

\subsection{Dimensions $1+n$ and $n+1$}

The first case, $1+n$ dimensions, is elementary. In this case $H^{+}$is a hyperboloid with two sheets and $N$ consists of a pair of points $\vec{x}$ and $\vec{y}$, one on each sheet. We can assume that $\vec{x}$ is a positive vector and $\vec{y}$ is a negative vector. The directed volume of $N$ is then

$$
\overrightarrow{\mathrm{Vol}} N=\vec{x}-\vec{y}
$$

which is the sum of two positive unit spacelike vectors $\vec{x}$ and $-\vec{y}$. It is elementary that the sum is shortest when they are parallel. (Indeed, if we switch space with time, this is the simplest case of the twin paradox in special relativity.) This is equivalent to the condition that $N$ is centered at the origin, the only thing to prove in this case.

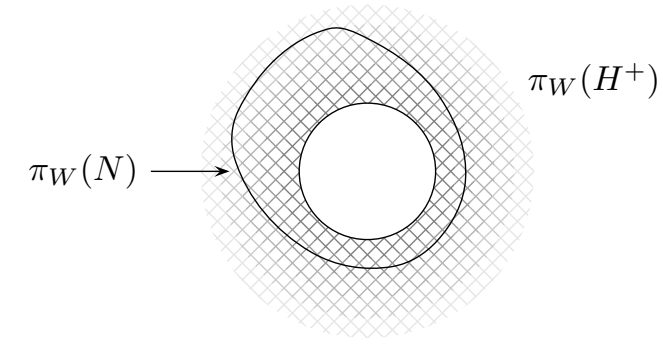

Figure 2: $\pi_{W}(N)$ rings the hole of $\pi_{W}\left(H^{+}\right)$

The second case, $n+1$ dimensions, is instructive for the last two cases, which are more difficult. Let $v_{n}$ be the volume of the unit ball in $\mathbb{R}^{n}$. Let $W$ be a spacelike $n$-plane passing through the origin and let

$$
S=W \cap H^{+}
$$

be the unit sphere in $W$. Let

$$
\pi_{W}: V \rightarrow W
$$

be the orthogonal projection onto $W$, and let

$$
\pi_{S}: H^{+} \rightarrow S
$$


be the radial projection onto $S$, generalizing the map $\pi^{+}$of Section 1.1. By the argument of Section 1.1, $\pi_{S}$, if restricted to $N$, is a homeomorphism. Equivalently, $\pi_{W}(N)$ is starlike. At the same time, $\pi_{W}\left(H^{+}\right)$is the complement of $S$. Consequently the area enclosed by $\pi_{W}(N)$ is at least $v_{n}$, the volume enclosed by $B$, because $\pi_{W}(N)$ must go around the hole in $\pi_{W}\left(H^{+}\right)$, as indicated in Figure 2 .

Thus for any spacelike $n$-plane $W$, the component of $\overrightarrow{\mathrm{Vol}} N$ which is orthogonal to $W$ is at least $v_{n}$. This implies that $\overrightarrow{\mathrm{Vol}} N$ is dual to a timelike vector. If we choose an orthonormal basis

$$
\vec{e}_{1}, \vec{e}_{2}, \ldots, \vec{e}_{n}
$$

of $W$ and extend with a postive orthogonal unit timelike vector $\vec{e}_{n+1}, \overrightarrow{\mathrm{Vol}} N$ becomes the monomial tensor

$$
\overrightarrow{\mathrm{Vol}} N=c \vec{e}_{1} \wedge \ldots \wedge \vec{e}_{n} .
$$

Moreover, $c \geq v_{n}$, so by computation in this basis,

$$
Q(\overrightarrow{\mathrm{Vol}} N) \geq v_{n}^{2}
$$

The point is that in a suitable basis for $V$, the only non-vanishing terms of $\overrightarrow{\mathrm{Vol}} N$ all have non-negative self inner product.

\subsection{Dimensions $n+2$ and $2+n$}

The third case, $n+2$ dimensions, requires a preliminary lemma about the exterior square $\bigwedge^{2} V$ interpreted as a Lie algebra:

$$
\bigwedge^{2} V \cong \operatorname{so}(V) \cong \operatorname{so}(n, 2) .
$$

Note that the first isomorphism is canonical, and that using this isomorphism,

$$
\langle X, Y\rangle=-\frac{1}{2} \operatorname{Tr}(X Y) .
$$

Among the elements of $\operatorname{so}(V)$ there are spacelike and timelike rotations. Since the timelike planes are all oriented, the timelike rotations can be divided into positive and negative. Also say that an element of $\mathrm{so}(V)$ is elliptic if it is a product of commuting spacelike and timelike rotations (positive or negative).

Lemma 1 (Paneitz) A convex combination of positive timelike rotations is elliptic. 
Here are some comments about the results and terminology of Paneitz [3, 4]. Among all convex cones in $\operatorname{so}(V)$ which are invariant under conjugation, there is a unique minimal closed cone $C_{0}$ and a unique maximal cone $C_{1}$ (necessarily closed). Define the infinitesimal angle $d>0$ of a rotation $R$ (either spacelike or timelike) by the relation

$$
\operatorname{Tr}\left(R^{2}\right)=2 d^{2} .
$$

Then according to Paneitz [3, page 340], the elements of $C_{0}^{\text {int }}$ are precisely those that are a commuting product of a positive timelike rotation by an angle $d_{0}$ and spacelike rotations by angles $d_{1}, \ldots, d_{k}$ (necessarily $2 k \leq n$ ) such that

$$
d_{0}>d_{1}+d_{2}+\ldots+d_{k} .
$$

Every timelike rotation is of this form (with $k=0$ ), hence any convex combination is as well.

Recall that an alternating $k$-tensor is simple if it is a wedge product of vectors. For a general quadratic form $Q$ on $V$ of signature $(a, b)$, say that a simple $k$-tensor in $\bigwedge^{k} V$ is spacelike (respectively timelike) if it is the wedge product of vectors that span a spacelike $k$-plane (resp. a timelike $k$-plane). A spacelike simple $a$-tensor (resp. a timelike simple $b$-tensor) is positive if its factors are positively ordered relative to the orientation of the $a$-plane (resp. the $b$-plane) they span. Recall that the Hodge star operator on $k$-tensors is defined as the unique linear operator

$$
*: \bigwedge^{k} V \rightarrow \bigwedge^{n+2-k} V
$$

such that

$$
*\left(\vec{e}_{1} \wedge \vec{e}_{2} \wedge \ldots \wedge \vec{e}_{k}\right)=\vec{e}_{k+1} \wedge \vec{e}_{k+2} \wedge \ldots \wedge \vec{e}_{n+2}
$$

for any positively oriented orthonormal frame

$$
\vec{e}_{1}, \vec{e}_{2}, \ldots, \vec{e}_{n+2} \text {. }
$$

We will need two facts about the Hodge star operator: first, that

$$
Q(* \omega)=(-1)^{b} Q(\omega)
$$

for any tensor $\omega$, and second that $\omega$ is a positive, spacelike, simple $a$-tensor if and only if $* \omega$ is a positive, timelike, simple $b$-tensor.

In terms of 2-tensors, Lemma 1 says that a convex combination of positive, timelike, simple 2-tensors can be expressed in the form

$$
d_{0} \vec{e}_{0} \wedge \vec{e}_{1}+d_{1} \vec{e}_{2} \wedge \vec{e}_{3}+\ldots+d_{k} \vec{e}_{2 k} \wedge \vec{e}_{2 k+1},
$$

where the vectors $\vec{e}_{0}, \vec{e}_{1}, \ldots, \vec{e}_{2 k+1}$ are orthonormal, and $\vec{e}_{0}$ and $\vec{e}_{1}$ are timelike. In addition if the pair $\left(\vec{e}_{0}, \vec{e}_{1}\right)$ forms a positive basis of the plane it spans, then 
$d_{0}$ is positive. We will need the dual statement that a convex combination of positive, spacelike, simple $n$-tensors can be expressed in the form

$$
\begin{aligned}
d_{0} *\left(\vec{e}_{0} \wedge \vec{e}_{1}\right) & +d_{1} *\left(\vec{e}_{2} \wedge \vec{e}_{3}\right)+d_{2} *\left(\vec{e}_{4} \wedge \vec{e}_{5}\right)+\ldots \\
& +d_{k} *\left(\vec{e}_{2 k} \wedge \vec{e}_{2 k+1}\right) .
\end{aligned}
$$

Finally, if $N$ is a neck, then $\overrightarrow{\mathrm{Vol}} N$ is realized as a convex combination of positive, spacelike, simple $n$-tensors by the obvious generalization of equation (2). Consequently $* \overrightarrow{\mathrm{Vol}} N$ can be expressed in the form of expression (6). If $W$ is a spacelike $n$-plane spanned by the vectors $\vec{e}_{2}, \ldots, \vec{e}_{2 k+1}$, then the projection of $N$ encloses a volume of at least $v_{n}$ by the idea illustrated in Figure 2. Thus

$$
d_{0} \geq v_{n},
$$

and

$$
Q(\overrightarrow{\mathrm{Vol}} N)=\sum_{i=0}^{k} d_{i}^{2} \geq d_{0}^{2} \geq v_{n}^{2}
$$

as desired.

Conjecture 4 is argued the same way in $2+n$ dimensions as in $n+2$ dimensions, except without the complication of applying Hodge duality.

\subsection{Trivial cases and open cases}

The case of $n+0$ dimensions is trivially true, since there is only one candidate for the neck $N$. The case of $0+n$ dimensions is vacuous.

The basic reason that the above arguments do not work in $a+b$ dimensions when both $a$ and $b$ are at least 3 is that the space of alternating $a$-tensors is bigger than the Lie group $\mathrm{SO}(a, b)$. Asymptotically

$$
\operatorname{dim} \bigwedge^{a} \mathbb{R}^{a+b}
$$

grows exponentially in $\min (a, b)$, while

$$
\operatorname{dim} \operatorname{SO}(a, b)
$$

grows quadratically. The general $a$-tensor does not admit an orthonormal basis such that all terms have positive energy. 


\section{Local stability}

In this section we argue that a flat neck is a local minimum of the energy $Q(\overrightarrow{\mathrm{Vol}} N)$ relative to the $C^{1}$ topology in $a+b$ dimensions.

Consider $\mathbb{R}^{a+b}$ together with the standard quadratic form $Q$ of signature $(a, b)$ given by

$$
Q(\vec{x}, \vec{y})=\vec{x} \cdot \vec{x}-\vec{y} \cdot \vec{y},
$$

using the standard dot products on $\mathbb{R}^{a}$ and $\mathbb{R}^{b}$. Let $\langle\cdot, \cdot\rangle$ be the associated bilinear form. Let $S^{a-1}$ be the standard unit $(a-1)$-sphere in the standard timelike $\mathbb{R}^{a} \subset \mathbb{R}^{a+b}$. The hyperboloid sheet $H^{+}$is perpendicular to $\mathbb{R}^{a}$ at the sphere $S^{a-1}$. Given a $C^{1}$ function

$$
\vec{f}: \mathbb{R}^{a} \rightarrow \mathbb{R}^{b}
$$

let $N$ be the set

$$
N=\left\{\left(\vec{x} \sqrt{1+f^{2}(\vec{x})}, \vec{f}(\vec{x})\right) \mid \vec{x} \in S^{a-1}\right\} .
$$

For suitable $\vec{f}, N$ is a neck, and every neck $N$ can be uniquely expressed in this form.

Let $\vec{e}_{1}, \ldots, \vec{e}_{a+b}$ be the standard basis of $\mathbb{R}^{a+b}$. Given a linear map

$$
L: \mathbb{R}^{a} \rightarrow \mathbb{R}^{b},
$$

we define an alternating $a$-tensor

$$
\Psi(L)=\sum_{k=1}^{a}(-1)^{k+1} L\left(\vec{e}_{k}\right) \wedge \vec{e}_{1} \wedge \ldots \wedge \widehat{\vec{e}_{k}} \wedge \ldots \wedge \vec{e}_{a} .
$$

In other words, $\Psi$ is the natural linear transformation

$$
\Psi: \operatorname{Hom}\left(\mathbb{R}^{a}, \mathbb{R}^{b}\right) \rightarrow \bigwedge^{a-1} \mathbb{R}^{a} \otimes \bigwedge^{1} \mathbb{R}^{b} \subset \bigwedge^{a} \mathbb{R}^{a+b}
$$

induced by the standard Hodge star operator on $\mathbb{R}^{a}$ and the standard dot product on $\mathbb{R}^{b}$. Using this notation, if $\vec{f}$ and its derivative $D \vec{f}$ are of order $\epsilon$, then

$$
\begin{aligned}
\overrightarrow{\operatorname{Vol}} N= & \left(v_{a-1}+\int a f^{2}(\vec{x}) d \vec{x}+o\left(\epsilon^{2}\right)\right) \vec{e}_{1} \wedge \ldots \wedge \vec{e}_{a} \\
& +\int \Psi(D \vec{f}(\vec{x})) d \vec{x}+o(\epsilon)
\end{aligned}
$$


Here all integrals are over the sphere $S^{a-1}$, as before $v_{a-1}$ is the volume enclosed by $S^{a-1}$, and the last term $o(\epsilon)$ consists of monomials with at least two wedge factors $\vec{e}_{k}$ with $k>a$. If we set

$$
Q[\vec{f}]=Q(\overrightarrow{\operatorname{Vol}} N)
$$

then the first variational derivative of $Q$ at $\vec{f}=0$ vanishes by symmetry, while the second variational derivative is given by

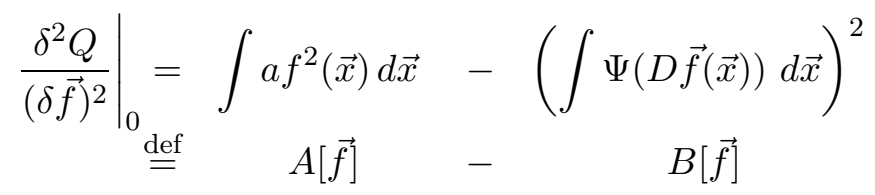

from equation (7). In the second line of equation (8), we define the functional $A[\vec{f}]$ to be the first term of the first line and the functional $B[\vec{f}]$ to be the second term.

We claim that the second variational derivative of $Q$ (equation (8)) is positive definite except for null directions given by the action of the symmetry group $\mathrm{SO}(a, b)$. These null directions correspond to the variations $\vec{f}$ which are linear. The general $\vec{f}$ has a harmonic expansion

$$
\vec{f}=\vec{f}_{0}+\vec{f}_{1}+\vec{f}_{2}+\ldots,
$$

where $\vec{f}_{k}$ is given by a degree $k$ polynomial which is orthogonal to lower-degree polynomials on the sphere $S^{a-1}$. The functional $A$ is proportional to the $L^{2}$ norm of $\vec{f}$ :

$$
A[\vec{f}]=a\|\vec{f}\|^{2}=a\left\|\overrightarrow{f_{0}}\right\|^{2}+a\left\|\overrightarrow{f_{1}}\right\|^{2}+a\left\|\overrightarrow{f_{2}}\right\|^{2}+\ldots .
$$

On the other hand, the functional $B$ is a quadratic function composed with the linear transformation

$$
\vec{f} \mapsto \int \Psi(D \vec{f}(\vec{x})) d \vec{x}
$$

This transformation is equivariant under $\mathrm{SO}(a) \times \mathrm{SO}(b)$, the stabilizer in $\mathrm{SO}(a, b)$ of the flat neck $S^{a-1}$. Its target is the irreducible representation $\bigwedge^{a-1} \mathbb{R}^{a} \otimes$ $\bigwedge^{1} \mathbb{R}^{b}$. Therefore it must annihilate all terms of the harmonic expansion of $\vec{f}$ except for $\vec{f}_{1}$, the sole term which lies in an isomorphic summand of the $L^{2}$ completion of the function space $C^{1}\left(S^{a-1}, \mathbb{R}^{b}\right)$. In other words,

$$
B[\vec{f}]=B\left[\vec{f}_{1}\right]=c|| \vec{f}_{1} \|^{2}
$$

for some constant $c$. This constant $c$ can be determined by noting that if $\vec{f}$ is linear, that is, $\vec{f}=\vec{f}_{1}$, then

$$
A[\vec{f}]-B[\vec{f}]=0
$$


because then $\vec{f}$ represents an infinitesimal motion of the neck given by the action of the Lie algebra $\operatorname{so}(a, b)$. Consequently $c=a$. Subtracting equation (10) from equation (9), we obtain

$$
\left.\frac{\delta^{2} Q}{(\delta \vec{f})^{2}}\right|_{0}=a\left\|\overrightarrow{f_{0}}\right\|^{2}+a\left\|\vec{f}_{2}\right\|^{2}+a\left\|\overrightarrow{f_{3}}\right\|^{2}+a\left\|\overrightarrow{f_{4}}\right\|^{2}+\ldots
$$

Thus the second variational derivative has the desired positivity property.

\section{Acknowledgements}

The author would like to thank Krystyna Kuperberg and Włodzimierz Kuperberg for corrections as well as Bruce Kleiner and Frank Morgan for their interest in the work.

\section{References}

[1] J Bourgain, V D Milman, New volume ratio properties for convex symmetric bodies in $\mathbb{R}^{n}$, Invent. Math. 88 (1987) 319-340

[2] G Kuperberg, A low-technology estimate in convex geometry, Internat. Math. Res. Notices, 1 (1992) 182-184

[3] S M Paneitz, Invariant convex cones and causality in semisimple Lie algebras and groups, J. Funct. Anal. 43 (1981) 313-359

[4] S M Paneitz, Determination of invariant convex cones in simple Lie algebras, Ark. Mat. 21 (1983) 217-228

[5] G Pisier, The Volume of Convex Bodies and Banach Space Geometry, Cambridge Tracts in Mathematics Volume 94, Cambridge University Press (1989)

[6] J Saint-Raymond, Sur le volume des corps convexes symétriques, from: "Initiation Seminar on Analysis, 20th Year: 1980/1981", (G Choquet, M Rogalsky, and J Saint-Raymond, editors) Exp. No. 11, 25. Univ. Paris VI, Paris (1981) 